 \\ (Official Journal of Mansoura Faculty of Medicine) \\ pISSN: 1110-211X; eISSN: 2735-3990
}

\section{Recent updates in Management of Borderline Ovarian Tumors}

\author{
Sara EIBayoumi ${ }^{1}$, Khalid Gaballah ${ }^{2}$, Ashraf Shoma ${ }^{3}$
}

\footnotetext{
${ }^{1}$ Final Year Medical Student, Mansoura Manchester Medical Programme, Faculty of Medicine, Mansoura University

${ }^{2}$ Lecturer of Surgical Oncology, Faculty of Medicine, Mansoura University

${ }^{3}$ Professor of Surgery, Faculty of Medicine, Mansoura University.
}

DOI: $10.21608 / \mathrm{mjmu} .2021 .83176 .1026$

Submit Date: 29-06-2021

Accept Date: 02-11-2021

Available online: $01-12-2021$

\section{Keywords}

- BOTs

- MPSC

- APST

\section{Abstract}

Borderline ovarian tumors (BOTs) need to be differentiated from ovarian carcinomas due to different presentation and management. Absent stromal invasion is the key differentiating feature on histopathological examination. Considering the degree of aggressiveness between epithelial ovarian carcinoma and benign ovarian neoplasms like cystadenoma, borderline neoplasms are closer to benign ovarian neoplasms as regards the clinical behavior. Surgery with intention of removal of all visible tumors is the most important pillar in treatment. Patients treated conservatively with fertility sparing surgery or laparoscopic surgery has low recurrence rate, making both options worth considering. Overall prognosis of these tumors is good with survival rate more than $90 \%$ at 10 years in early stage borderline tumors. However, small percentage of patients with borderline neoplasms may show more aggressive form of the disease, and trials have been made to figure out the histological correlates that might predict for worse outcome. There is no added advantage of postoperative adjuvant chemotherapy or radiation in any stage especially with non-invasive component. Follow up is done by observation of the patient and any further intervention is kept reserved for recurrent disease.

Corresponding author: Sara ElBayoumi Mohamed Abodabesh, Student, Faculty of Medicine, Mansoura University Phone: 01154888196. Email: saraelbayoumi7@gmail.com 
Borderline ovarian tumors are characterized by certain criteria that includes having atypical epithelial proliferation with lack of stromal invasion histologically. [1] BOT's name was firstly suggested by Taylor in 1929 as "semimalignant" disease because BOTs have certain characteristics that combine both being benign and malignant ovarian tumors, but the prognosis of the patient who has BOT is better than any other ovarian tumor even with peritoneal involvment. ${ }^{[2]}$ In 1961, FIGO (The International Federation of Gynecology and Obstetrics) described BOTs as a unique category due to the fact that they have a low malignant inclination and so The World Health Organization (WHO) named them "borderline" in 1973. At the present time, BOTs are described by three names: borderline tumor, tumor of low malignant potential, and atypical proliferative tumor (Figure1) $^{[3]}$.

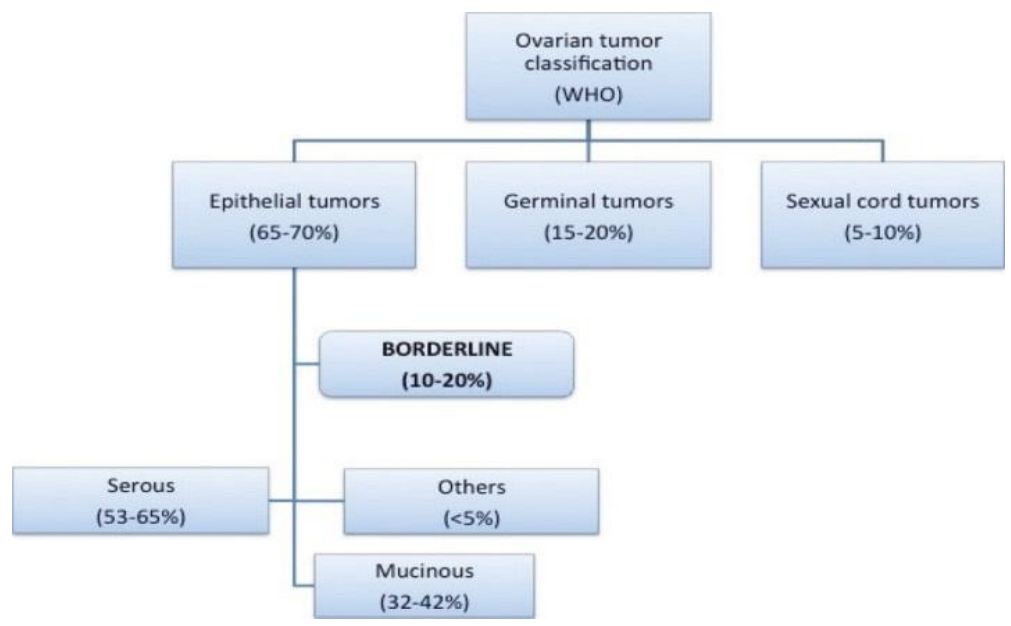

Figure 1: Ovarian tumour classification $(\mathrm{WHO})^{[3]}$

Borderline ovarian tumors are different from epithelial ovarian cancer in that they have low incidence, is not particularly associated with abnormalities in BRCA genes, rate of survival is higher due to early stage diagnosis, and associated with infertility which occurs on many occasions. One of the most important things to take care of in these patients is the protection of fertility as it most commonly occurs in young women. The way to manage the treatment of these tumors is still not yet defined and debatable. The most recent researches draw a line under the staging of these tumors by radical or conservative surgery and according to the staging, decide the best option of treatment and acquire a precise prognosis ${ }^{[4]}$.

\section{Incidence:}

Borderline ovarian tumors make up about $20 \%$ of the ovarian tumors. In author's experience, the patients with BOTs who were treated over the last 21 months made up only $9.3 \%{ }^{[5]}$.

\section{Epidemiology:}

The risk of BOT is increased by primary infertility and nulliparity. However, 
breastfeeding, multiple pregnancies and oral contraceptives are protective ${ }^{[6]}$.

There are several hypotheses that have been outlined to support the reproductive risk factors. If repeated microtrauma occurred to ovarian surface during ovulation, it would eventually lead to ovarian malignancy. On the other hand, another hypothesis mentions that malignancy can happen after ovarian exporsure to increased levels of gonadotropins. A few case control studies observed a two to four folds increase in the risk of BOT when the patients take fertility drugs followed by ovarian stimulation and multiple ovarian punctures. According to the hormonal hypothesis, estrogen and androgen stimulates neoplasms growth, meanwhile progesterone acts as a protective factor. Another hypothesis denotes the interrelation between endometriosis and external carcinogens (as talc, asbestos) in tumorigenesis especially clear cell and endometrioid type of BOTs (Figure 2) ${ }^{[7]}$.

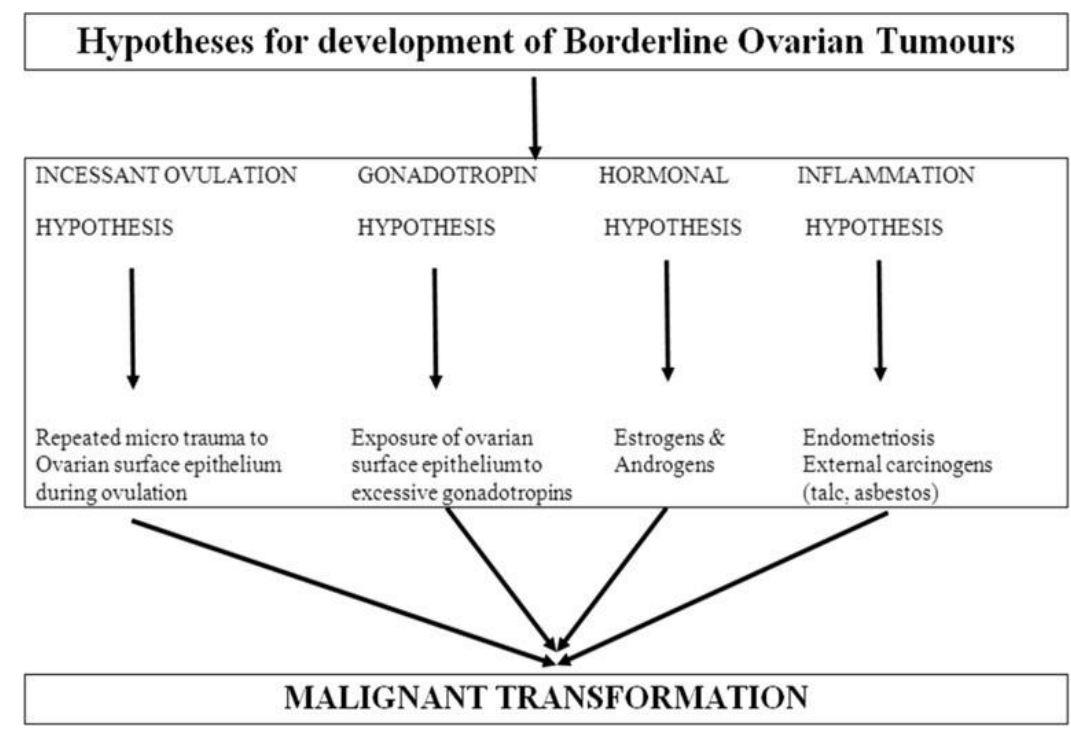

Figure 2: Hypotheses for development of borderline ovarian neoplasms ${ }^{[7]}$.

Women who underwent in-vitro fertilization (IVF) have enhanced risk of being diagnosed with borderline ovarian neoplasms. In addition, women with BRCA mutations are hardly ever seen with borderline ovarian tumors. Almost 10$35 \%$ of patients with BOT are infertile ${ }^{[8]}$. Serum markers are not helpful in diagnosing borderline ovarian neoplasms, except for advanced-stage BOT. $83 \%$ of patients with advanced stage disease and $40 \%$ of women with stage 1 borderline ovarian tumors were observed with unusual levels of CA $125^{[9]}$.

\section{Histopathology and Classification}

BOTs are classified based on the FIGO classification, which is almost similar to that of ovarian tumors. However, the majority of BOTs $(60-70 \%)$ are diagnosed at stage 1 in contrast to ovarian tumors which only $25 \%$ of them are diagnosed at stage 1 . It is very rare to find BOTs 
in late stages as 2 or 3 , moreover exceptional at stage $4 .{ }^{[10]}$ In terms of histopathology, there are important features that have to be distinguished for BOT differentiation from benign cyst adenoma; these features are: existence of epithelial budding, multilayered epithelium, enhanced mitotic activity, presence of nuclear atypia $^{[10]}$.

In addition, the difference between BOT and ovarian cancer is the absence of stromal invasion. The majority of borderline epithelial tumors, like that of carcinomas, are serous tumors (53-65\%). Mucinous BOT makes up between $32 \%$ and $42 \%$ of the total. The rest of BOTs types also includes endometrial tumors, clear cell tumors, Brenner's tumors which make up less than $5 \%(\text { Table } 1)^{[11]}$.

Table 1. Histological classification of borderline ovarian neoplasms. ${ }^{[11]}$

\begin{tabular}{|c|c|c|c|}
\hline Type & Subtype WHO & Blaustein & Other histological characteristics \\
\hline Serous & $\begin{array}{l}\text { Typical subtype } \\
(90 \%) \\
\text { Micropapillary } \\
\text { subtype }(10 \%)\end{array}$ & $\begin{array}{l}\text { Benign group, APSTs } \\
\text { Low grade malignant group: } \\
\text { Noninvasive MPSC } \\
\text { Low grade malignant group: APST } \\
\text { with invasive peritoneal implants }\end{array}$ & $\begin{array}{l}\text { a) Usual type (eosinophilic type), which } \\
\text { are cells with ample eosinophilic } \\
\text { cytoplasm } \\
\text { b) structures are identical in appearance } \\
\text { to invasive lowgrade micropapillary } \\
\text { serous carcinoma }\end{array}$ \\
\hline Mucinous & $\begin{array}{l}\text { Intestinal subtype } \\
(85 \%) \\
\text { Mullerian subtype } \\
(15 \%)\end{array}$ & $\begin{array}{l}\text { Intestinal subtype }(85 \%) \\
\text { Mullerian subtype }(15 \%)\end{array}$ & $\begin{array}{l}\begin{array}{l}\text { Mucinous borderline tumor with } \\
\text { microinvasion and/or intraepithelial } \\
\text { carcinoma }\end{array} \\
\begin{array}{l}\text { Microinvasion and/or intraepithelial } \\
\text { carcinoma as well as peritoneal implants, may } \\
\text { be present. }\end{array}\end{array}$ \\
\hline Endometriod & $\begin{array}{l}\text { Adenomatos } \\
\text { appearance } \\
\text { Glandular/papilla } \\
\text { ry appearance }\end{array}$ & & $\begin{array}{l}\text { Endometrioid borderline tumor with } \\
\text { microinvasion and/ or intraepithelial } \\
\text { carcinoma and/or extraovarian implants }\end{array}$ \\
\hline Clear cell & & & $\begin{array}{l}\text { Clear-cell borderline tumor with microinvasion } \\
\text { and/or intraepithelial carcinoma }\end{array}$ \\
\hline $\begin{array}{l}\text { Brenner } \\
\text { (transitional cell) }\end{array}$ & & & $\begin{array}{l}\text { Resembling low-grade papillary urothelial } \\
\text { carcinoma of the urinary tract }\end{array}$ \\
\hline
\end{tabular}

\section{Serous BOT}

WHO studies of ovarian tumors showed that there are two types of serous borderline ovarian tumors: typical serous BOTs (90\%) and micropapillary patters of BOTs (5-10\%). However, recent studies showed that serous
BOTs can be further classified into several categories, because they had several different biological potentials. (Table 1) ${ }^{[12]}$.

The first type is typical pattern, which makes up $90 \%$ of the cases and have unilocular cystic mass with fine interior septa. (Figure 3$)^{[13]}$. The second type is micropapillary pattern found in 
$10 \%$ of the cases and presenting specific more invasive form, greater prevalence of histological features. They have a worse bilateralilty and upstaging after performing prognosis as compared to typical pattern serous restaging surgery ${ }^{[13]}$.

BOTs because of increased rate of recurrence,

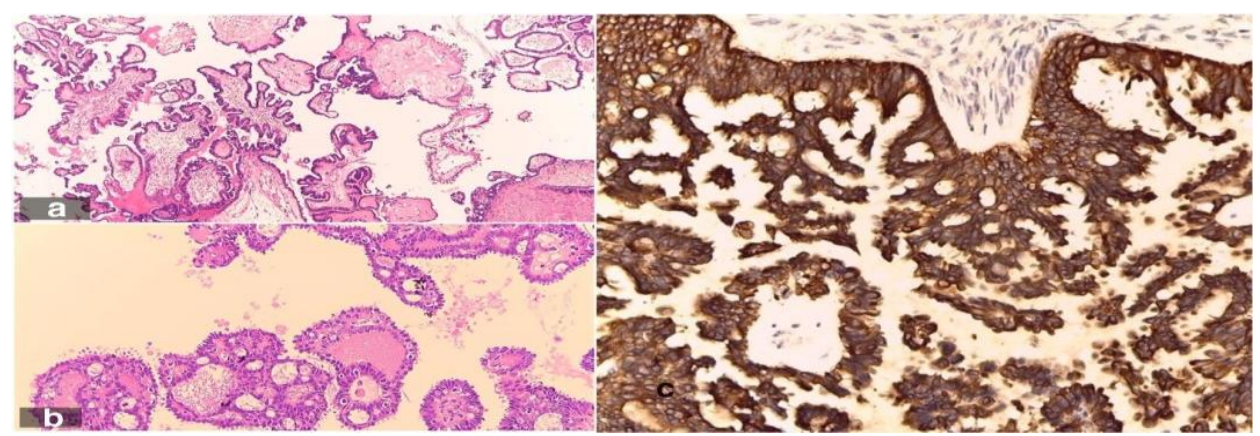

Figure 3: Low power view of a serous borderline ovarian tumor with numerous papillae, broad and edematous, with complex, hierarchical branching (a) small papillae which appear detached from larger papillae with epithelial proliferation (b) immunostaining demonstrating a diffusely and strongly positive reaction (c) ${ }^{[13]}$.

\section{Mucinous BOT}

They appear to be larger in size compared with serous BOT and on gross examination they have either unilocular or multilocular cyst with fine interior septa and intramural nodules.(Figure 4) Peritoneal implants are not common (15\%) and if they occur, pseudomyxoma peritonei must be excluded. It is considered a differentiated as peritoneal involvement of mucinous carcinoma is originally from digestive origin, most probably the appendix ${ }^{[14]}$. There are two sub types: Intestinal (85-90\%): The most common type and the majority is unilateral. However, primary intestinal cancer must be excluded in presence of bilateral involvement. [14] Endocervical or müllerian (10-15\%): they are bilateral in $40 \%$ of cases and $20-30 \%$ of which are related to pelvic endometriosis, ipsilateral endometriomas or BOT of mixed histology (seromucinous) ${ }^{[14]}$.

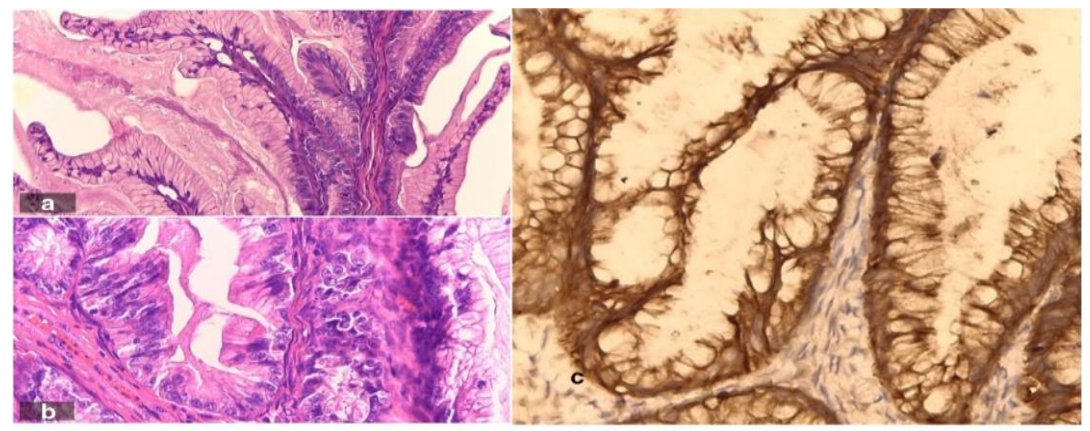

Figure 4: High power view of a mucinous borderline ovarian tumor that shows papillae with scanty stromal cores (a) lined by atypical stratified cells (b) and immunostaining that shows a diffusely and strongly positive reaction (c) [14] 


\section{Pathogenesis:}

There are two pathways which have been suggested in pathogenesis of serous BOTs. The first one is "low grade" pathway which includes BRAF and KRAS mutations, where the serous ovarian cyst adenomas advance to serous BOTs which will further be low grade serous epithelial cancer through a continuation of histologic precursor lesions. Yet only $2 \%$ of the serous borderline ovarian tumors become carcinomas through the "low grade" pathway ${ }^{[15]}$. The second pathway is the "high grade" pathway which includes p53 gene mutations. There is no known precursor, but most of the serous ovarian cancers belong to the high-grade pathway ${ }^{[15]}$.

Serous borderline ovarian tumors are distinguished via activation of specific tumor suppressor genes (SERPINA 5 and dual specificity phosphatase 4) which hinder the breaking down of the extracellular matrix, an important incident in pathogenesis of invasive growth. ${ }^{[16]}$ Mucinous tumorigenesis includes a pattern of malignant modifications from benign mucinous neoplasms to carcinomas. There are three classes of bas oncogenes which are $\mathrm{K}, \mathrm{N}$ and $\mathrm{H}$. Mucinous BOTs had an increased rate of KRAS mutations when compared to that of muchness cyst adenoma, yet less than that of mutinous carcinoma. It is not evident if BRCA1 and BRCA2 multiplies the chances of BOT ${ }^{[16]}$. Endometrioid borderline ovarian neoplasms, in contrast to serous and mucinous BOTs, are identified by mutations including beta catenin gene $(50 \%)$, PTEN gene $(20 \%)$ as well as micro satellite instability gene (up to $50 \%)^{[17]}$.

\section{Clinical Presentation:}

The majority of patients having BOT are asymptomatic. It can be accidently discovered during pelvic examination as a pelvic mass, or ovarian masses can be detected by screening for abdominal ultrasound. About the majority percent as $50-60 \%$ present with symptoms as abdominal pain or discomfort, abdominal swelling, irregular GUT motions, persistent tiredness, or weight loss. A minority of women $(10 \%)$ have irregular uterine bleeding ${ }^{[18]}$.

\section{Diagnosis:}

BOTs are hard to discover clinically until they have very large size or in very advanced late stages. Pelvic ultrasound can help detect the ovarian masses, but it is not specific or sensitive, so it is not such a helpful screening tool to use for all patients (Figure 5). BOTs appear on ultrasound typically as: unilocular cyst with solid papillary projections (defined as any projection with a height $\geq 3 \mathrm{~mm}$ ) arising from the inner wall and with a positive ovarian crescent sign (Figures 6,7). In contrast to ovarian carcinoma, BOTs do not present with ascites. Serum CA 125 also is not specific or accurate for diagnosing or following up with a patient having borderline ovarian tumor. In a systematic review, $53.8 \%$ of patients had negative CA125 concentrations ${ }^{[19]}$.

The diagnosis of BOTs is done intra-operative through frozen section examination of the tumor or post-operatively. The criteria for diagnosing 
include absence of stromal invasion and two or more of the following criteria: epithelial papillary projections, cellular pleomorphism, nuclear atypia as well as mitotic activity ${ }^{[19]}$. proliferation, stratified epithelium, microscopic

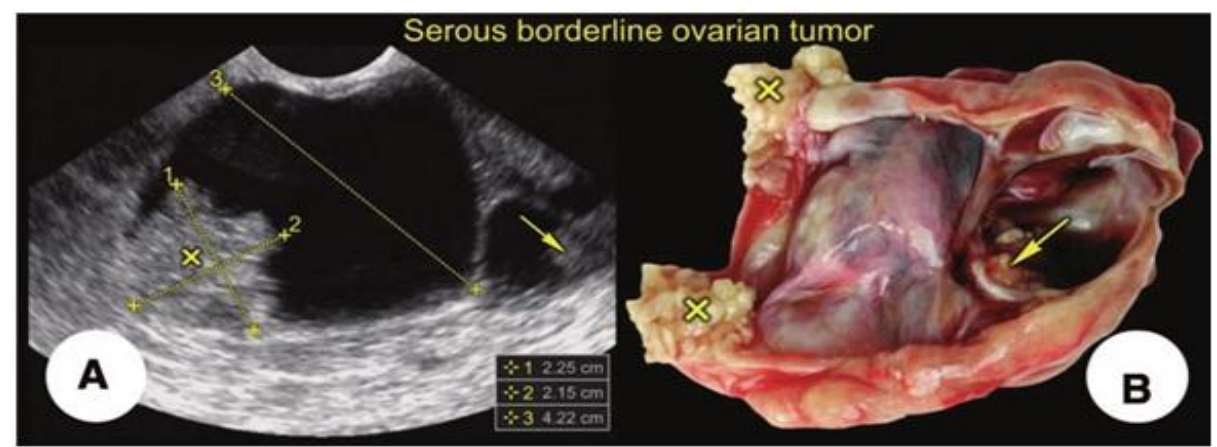

Figure 5: Serous borderline tumor (transvaginal scan). Multilocular-solid tumor with papillae, rather smooth inner cyst wall, and regular septa and anechoic intracystic fluid ${ }^{[19]}$.

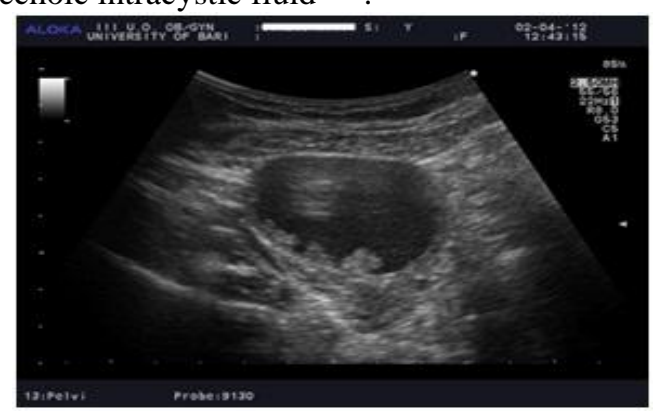

Figure 6: Unilocular cyst with solid papillary projections in BOT ${ }^{[19]}$.

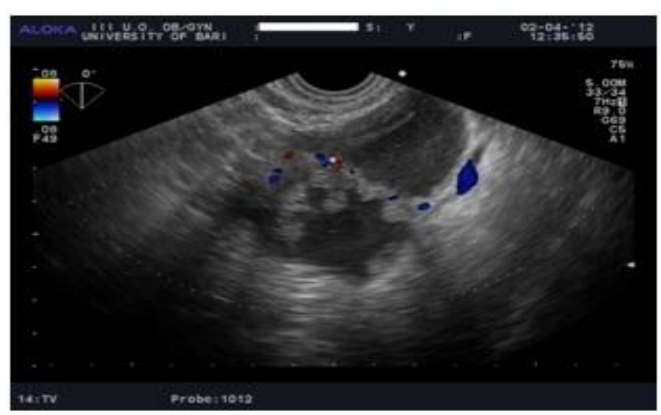

Figure 7: Papillary projections in BOT ${ }^{[19]}$.

\section{Prognosis:}

Usually BOTs have good prognosis, with the exception of $10 \%$ recurrence rate and $20-30 \%$ malignancy risk. The characteristics that appear to be associated with poor prognosis are: Transformation of borderline tumors to invasive disease which is dependent on: cell type, stage, implant type (for serous BOTs), micropapillary architecture (for serous BOTs) and is linked to invasive implants in $45 \%$ of patients, microinvasion [20]. Postoperative macroscopic residual disease, pathologically advanced stage, and extra-ovarian invasive implants in addition to pathologically advanced stage are major predictor for both recurrences and poorer survival. Histological type: Serous tumors have 
poor prognosis in contrast to non-serous tumors, which have excellent prognosis irrespective of the presence or absence of intraepithelial carcinoma and/or microinvasion [21]. Genetic factors: Presence of aneuploid DNA content have a poor prognosis regarding recurrence and survival. Both BRAF and KRAS mutations in serous and only KRAS mutations in mucinous tumor have been linked to progression and worse prognosis [21]. Type of surgery and surgical approach affect the prognosis by having an impact on residual disease and in presence of residual tumor load have poor impact on recurrence rate ${ }^{[20]}$.

Conservative treatment in form of preservation of uterus and one ovary, is associated with increased disease recurrence in the remaining ovary, although it does not lead to poorer survival as most of the recurrences in the remaining ovary are noninvasive type especially for early stages. Unilateral oophorectomy rather than cystectomy is preferred when the contralateral ovary is normal in younger age patients where fertility is desired so as to avoid recurrence. Conservative laparoscopic approach is associated with twice more recurrence rate than laparotomy, but overall survival rate is not reduced ${ }^{[21]}$.

\section{Postoperative adjuvant treatment}

(chemotherapy, radiotherapy) is not recommended as it leads to increased treatment related morbidity and mortality rather than the disease itself ${ }^{[21]}$.

\section{Therapeutic approaches:}

The main therapy for borderline ovarian neoplasms is surgical resection. There has been a shift in management from doing radical surgery to a more conservative approach, as BOT has a tendency to influence younger females ${ }^{[22]}$.

As the diagnosis of BOT is determined preoperatively, intra-operative frozen section examination is important to treat a suspicious ovarian mass to customize the extent of resection. However, it must be noted that frozen section has a limited accuracy, it certainly depends on the experience of the histopathologist. BOT is under-diagnosed in intra-operative frozen section in $31 \%$ of patients. A sub-diagnosis differentiates a BOT from malignant tumor in $25 \%$ of patients, which means that the patient has to get through another surgery and the final histopathological results appear to be malignant ovarian tumor ${ }^{[22]}$.

\section{A- Radical surgery}

The standard radical surgery should be performed to women who has completed their reproductive wishes, which is total hysterectomy with bilateral salpingooophorectomy, inframesocolic omentectomy, resection of macroscopically suspicious lesion and peritoneal washing, including exploration of the abdominal cavity. Nodal involvement does not particularly affect the survival of the patients, in spite of the fact that it is associated with an increased rate of recurrence, so pelvic and paraaortic lymphadenectomy is not an important 
requirement as it does not have a prognostic value in BOT. Involvement of the lymph nodes does not reduce survival; neither does lymphadenectomy enhance it. Appendectomy should be done to patients who have abnormal gross appearance of the appendix especially with the mucinous type to rule out synchronous or primitive appendiceal neoplasm. (Figure 8) ${ }^{[23]}$.

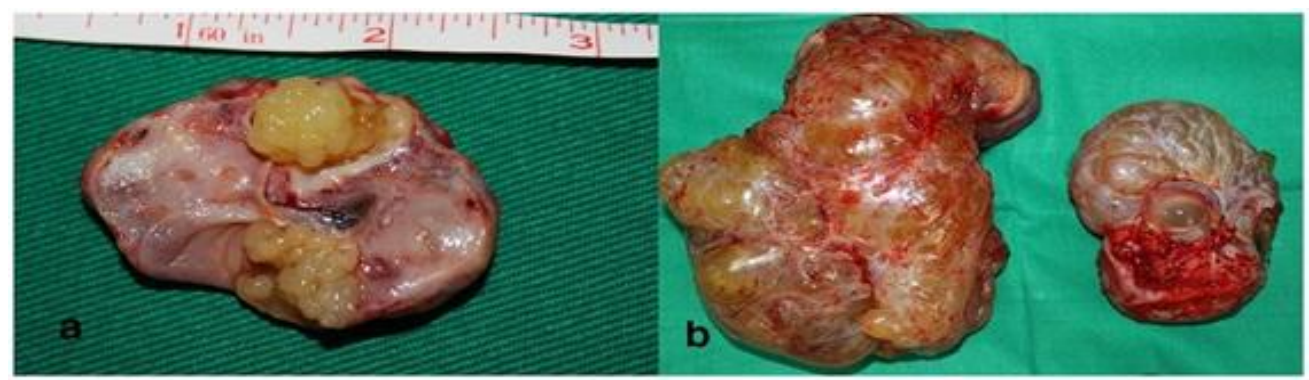

Figure 8: Surgical specimen of an ovary. a Papillary serous ovarian cystadenoma. b Bilateral mucinous ovarian cystadenoma ${ }^{[23] .}$

\section{B- Conservative surgery}

Since the majority of the patients with BOT are in their childbearing age, fertility preservation is a critical concern in deciding treatment of borderline ovarian tumors. Fertility sparing surgical technique, which preserves uterus and at least part of one ovary, may be the ideal treatment especially for patients with FIGO stage 1 and age less than 40 years. Therefore, if tumor is limited to the ovary in patients, unilateral salpingo-oopherectomy or ovarian cystectomy with complete surgical staging is recommended. However, biopsy of the contralateral ovary should be avoided, because it can result in peritoneal adhesions or unwanted damage to the ovarian reserve. Biopsy should be done only in patients with bilateral ovarian involvement, cystectomy in unilateral or bilateral ovaries, or unilateral salpingooophrectomy with contralateral cystectomy. Although fertility sparing treatments for BOTs may allow future pregnancies, it also carries and increased risk of recurrence. As a result, the gynecologic oncologist has to consider the advantages and disadvantages of a fertility sparing approach in females having BOTs and counsel the female on the importance of the long-term follow up ${ }^{\text {[24]. }}$

There are two types of conservative surgery; the first one is unilateral salpingo-oopherectomy (USO) and the second is unilateral ovarian cystectomy with or without contralateral ovarian cystectomy (ultra-conservative surgery). The higher rate of relapse is associated with cystectomy compared to USO ${ }^{[24]}$.

For serous tumors, several factors should be considered as the age of the patient, persistence of normal ovarian tissue on ultrasonography or MRI, and antra follicle count. For mutinous tumors, although the lesions are unilateral, they are often accompanied with invasive lesions, so the conservative management by cystectomy is not necessary ${ }^{[25]}$.

Bilateral salpingo-oopherectomy is preferred in patients with massive BOT, as preservation of 
any part of the ovary is not practical. The uterus could be preserved for oocyte donation or transfer of frozen embryos obtained before the bilateral salpingo-oophorectomy ${ }^{[25]}$.

Complete resection should be done for serous BOTs, as the aggressive nature and poor prognosis of invasive peritoneal implants may increase the risk to progression to invasive carcinoma. Mucinous tumors are more probably to turn out into invasive carcinoma ${ }^{[25]}$.

\section{Completion of surgery after fertility-sparing approach}

The recurrence rates of BOTs are higher after fertility sparing surgery than following radical surgery; nevertheless, after the desire for conception is completed, whether there must be a second surgical look to remove the uterus and contra-lateral ovaries is still controversial. Studies showerd that there is no difference in means of survival rates after surgery for BOTs ${ }^{[26]}$. It was suggested that radical surgery should be done for serous BOTs in case of disease recurrence. Whereas, surgery completion should be done for mucinous BOTs because many mucinous BOTs relapse as invasive ovarian carcinomas. So, in these young women, the psychological impact on them is very important and must not be ignored, hence an accurate preoperative fertility counseling should be done [26].

\section{Laparoscopy for BOTs}

During this era, benign ovarian masses are often dealt with via laparoscopy. The role of laparoscopic surgery for BOTs is still indefinite, yet there are advantages such less adhesion risk, less morbidity, shorter hospital stay, shorter post-operative recovery and better cosmoses. However, there are a few disadvantages as it carries a higher risk of rupture to the cyst capsule and incomplete staging occur more commonly with laparoscopy than by laparotomy [27].

\section{Re-staging Surgery}

In BOTs patients who undergone oophorectomy or cystectomy should have no any additional staging surgery, but regular transvaginal ultrasonography follow up is important. Studies noting the similarity and differences between complete surgical staging (CSS) with incomplete surgical staging in women with BOTs showed that restaging must be individualized and be supported by doctor's opinion and woman's wish. They came to an agreement that restaging surgery may be suggested if: ${ }^{[28]}$

(1) There are histological criteria implying invasive recurrence (peritoneal implant or micropapillary pattern).

(2) Peritoneum has not been clearly reported as "normal" or if there was no systematic exploration within the initial operation.

(3) If macroscopic peritoneal implants have been found intraoperatively.

(4) If gross lesion exists following initial operation. 
(5) If the patient is less likely to return for normal follow-up. Complete surgical staging was reduced to a decreased recurrence risk, although a non-significant correlation with survival was detected ${ }^{[28]}$.

Generally, BOT women must be closely followed up after the conservative surgery, therefore the advantages and disadvantages should be assessed by gynecological oncologist. Balancing the treatment with the oncological safety in mind, remains a challenging task over the last decades ${ }^{[28]}$.

\section{Follow up}

Follow-up should be done every three months during first two years, every six months between 2 - 5 years and then annually. Clinical evaluation must be added to TVS and CA 125 concentrations. A detailed follow-up of patients who were treated conservatively, with an attention to the remaining ovary, is a must. MRI should be done if local recurrence is suspected while a contrast enhanced CT is useful if there is suspicion of extrapelvic recurrence ${ }^{[29]}$.

Adjuvant chemotherapy (cisplatin-based) is not needed in stage 1 BOTs and debatable in advanced-stage BOTs. The role of hormonotherapy in the treatment of BOTs is still controversial, even though $>90 \%$ of BOTs are estrogen receptor positive. Any contraceptive methods including hormonal contraceptives and hormone replacement therapy can be used safely in the patients with a diagnosis of BOT ${ }^{[29]}$.

\section{Management of recurrence}

The site of recurrence is most common in the remaining ovary. The only situation where recurrent disease could affect the prognosis is when the character of the recurrent disease is invasive. Cytoreductive surgery should be performed when an extra ovarian borderline or invasive relapse occurs. Cytoreductive surgery is the best as it acts as an independent prognostic factor and will determine the overall survival. A study revealed a mortality rate in women with optimal debulking of $12 \%$, compared with $60 \%$ of women who were suboptimally debulked ${ }^{[30]}$.

\section{Conclusion}

BOTs differ from the epithelial ovarian malignancies in their excellent prognosis, curability with surgery, and being seen in relatively young ages. Thus, fertility sparing, and conservative surgical approaches are currently highly endorsed. Preoperative diagnosis of BOTs may be challenging as the clinical and ultrasonographic features might overlap with invasive carcinomas and sometimes with benign adnexal masses. Frozen section is necessary for the intraoperative diagnosis, despite the fact that the diagnostic value of frozen section is not as elevated as in invasive ovarian carcinomas. Conservative approach might improve the recurrence rate without worsening the general survival. The definitive role of laparoscopic surgery with its pros and cons within the treatment of BOTs has to be further studied. 


\section{References:}

1. Prat J.(2017).Pathology of borderline and invasive cancers. Best practice $\&$ research Clinical obstetrics \& gynaecology; 41:1530.

2. Carcangiu ML, Kurman RJ, et al. (2014). WHO classification of tumours of female reproductive organs: International Agency for Research on Cancer.

3. Karlsen NMS, Karlsen MA, et al.(2016). Relapse and disease specific survival in 1143 Danish women diagnosed with borderline ovarian tumours (BOT). Gynecologic oncology;142(1):50-3.

4. Colombo N, Sessa C, et al. (2019). ESMOESGO consensus conference recommendations on ovarian cancer: pathology and molecular biology, early and advanced stages, borderline tumours and recurrent disease. Annals of Oncology;30(5):672-705.

5. Tumor-An BO, Gyn O. (2019). Borderline Ovarian Tumor-An Overview and Evidence Based Management. Pan;2(1):306.

6. Nayyar N, Lakhwani P, et al. (2017). Management of Borderline Ovarian Tumors-Still a Gray Zone. Indian Journal of Surgical Oncology ;8(4):607-14.

7. Zhang Y, Li C, Luo S, et al. (2020). Retrospective Study of the Epidemiology, Pathology, and Therapeutic Management in Patients With Mucinous Ovarian Tumors.
Technology in cancer research \& treatment; 19:1533033820946423.

8. Sun Y, Xu J, et al. (2020). The Diagnosis, Treatment, Prognosis and Molecular Pathology of Borderline Ovarian Tumors: Current Status and Perspectives. Cancer Management and Research; 12:3651-9.

9. Gica N, Mustata L, et al. (2020). Management of Borderline Ovarian Tumors: Series of Case Report and Review of the Literature. Indian Journal of Surgery; 12:1-8.

10. Hada T, Miyamoto M, et al. (2020). Ovarian Seromucinous Borderline Tumors Are Histologically Different from Mucinous Borderline Tumors. In Vivo;34(3):1341-6.

11. Genestie C, Auguste A, et al. (2020). Histological classification of mucinous ovarian tumors: inter-observer reproducibility, clinical relevance, and role of genetic biomarkers. Virchows Archiv; 3:1-7.

12. Zheng R, Heller DS. (2019). Borderline Brenner tumor: a review of the literature. Archives of pathology \& laboratory medicine;143(10):1278-80.

13.Hauptmann S, Friedrich K, et al. (2017). Ovarian borderline tumors in the 2014 WHO classification: evolving concepts and diagnostic criteria. Virchows archiv;470(2):125-42.

14. Chen RF, Tao X, et al. (2020). Mucinous borderline ovarian tumors with and without Intraepithelial Carcinoma: Differences in 
clinicopathologic features and fertility results. Journal of Obstetrics and Gynaecology Research;46(4):646-53.

15. Lu J, Pi S, et al. (2019). Value of normalized apparent diffusion coefficients in differentiating between borderline and malignant epithelial ovarian tumors. European journal of radiology; 118:44-50.

16. Seong SJ, Kim DH, et al. (2015). Controversies in borderline ovarian tumors. Journal of gynecologic oncology;26(4):3439.

17. Gungorduk K, Asicioglu O, et al. (2017). The impact of surgical staging on the prognosis of mucinous borderline tumors of the ovaries: a multicenter study. Anticancer research;37(10):5609-16.

18. Sozen H, Vatansever D, et al. (2019). Clinicopathological analysis of borderline ovarian tumours and risk factors related to recurrence: experience of single institution. Journal of Obstetrics and Gynaecology;39(2):253-8.

19. Shah JS, Mackelvie M, et al. (2019). Accuracy of intraoperative frozen section diagnosis of borderline ovarian tumors by hospital type. Journal of minimally invasive gynecology.;26(1):87-93.

20. Jia SZ, Xiang Y, et al. (2020). Oncofertility outcomes after fertility-sparing treatment of bilateral serous borderline ovarian tumors: results of a large retrospective study. Human Reproduction;35(2):328-39.
21. Jiao X, Hu J, Zhu L. (2017). Prognostic factors for recurrence after fertilitypreserving surgery in patients with borderline ovarian tumors: a systematic review and meta-analysis of observational studies. International Journal of Gynecologic Cancer ;27(9).

22. Gica N, Mustata L, et al. (2020). Management of Borderline Ovarian Tumors: Series of Case Report and Review of the Literature. Indian Journal of Surgery:1-8.

23. Zhao J, Liu C, et al. (2018). Short-term outcomes and pregnancy rate after laparoscopic fertility-sparing surgery for borderline ovarian tumors: a single-institute experience. International Journal of Gynecologic Cancer ;28(2).

24. Maramai M, Barra F, et al. (2020). Borderline ovarian tumours: management in the era of fertility-sparing surgery. ecancermedicalscience; 14.

25. Helpman L, Yaniv A, et al. (2017). Fertility preservation in women with borderline ovarian tumors-how does it impact disease outcome? A cohort study. Acta Obstetricia et Gynecologica Scandinavica ;96(11):1300-6.

26. Sleiman Z, Karaman E, et al. (2019). Fertility preservation in benign gynecological diseases: current approaches and future perspectives. Journal of Reproduction \& Infertility;20(4):201. 
27. Morgan RJ, Alvarez RD, et al. (2013).

Ovarian cancer, version 2.2013. Journal of the National Comprehensive Cancer Network;11(10):1199-209.

28. Abascal-Saiz A, Sotillo-Mallo L, et al. (2014). Management of borderline ovarian tumours: a comprehensive review of the literature. ecancermedicalscience $; 8$.

29. Prat J. (2014). The results of conservative (fertility-sparing) treatment in borderline ovarian tumors vary depending on age and histological type. European Society for Medical Oncology.

30. Gouy S, Maria S, et al. (2020). Results After Conservative Surgery of Stage II/III Serous Borderline Ovarian Tumors. Annals of Surgical Oncology:1-8. 